\title{
Tumor de Krukenberg de origen colónico
}

\author{
Krukenberg tumor of colonic origin
}

\author{
Carlos Enrique Soto Aburto, * Mario Martínez Ruiz, ${ }^{\ddagger}$ \\ Elvira Márquez Aguirre, ${ }^{*}$ Luis Ramón Pérez Medrano ${ }^{\S}$
}

\section{Resumen}

El tumor de Krukenberg es un fibroma de origen digestivo y puede presentar metástasis hacia el ovario; su mayor incidencia ocurre entre los 30 y 40 años. De entre los tumores malignos del ovario, un 3 al $6 \%$ corresponde a tumores metastásicos. Una de sus principales características es el crecimiento rápido; aproximadamente el 60 a $80 \%$ se presentan de manera bilateral. Microscópicamente demuestra material mucoso abundante en forma de anillo de sello. El adenocarcinoma de colon es el tumor que mayormente simula al carcinoma primario de ovario; del 10 al $30 \%$ de las mujeres con carcinoma de colon presentará algún foco tumoral secundario en ovario. Caso clínico: Paciente femenino de 41 años con dolor abdominal generalizado y vómito; se le realizó un ultrasonido y se encontró una masa de $11 \mathrm{~cm}$ dependiente del ovario izquierdo. También se realizó una laparoscopia que permitió visualizar líquido verde y abundante en cavidad; implantes peritoneales en vejiga, fondo de saco y epiplón; se tomó líquido y nódulo de epiplón y se enviaron a transoperatorio, el cual reporta adenocarcinoma metastásico. Se procedió a realizar una laparotomía con la finalidad de citorreducción. El reporte histopatológico definitivo fue: adenocarcinoma moderadamente diferenciado primario de colon, metastásico a ovario o tumor de Krukenberg. Actualmente la paciente continúa su tratamiento a base de quimioterapia en el servicio de oncología. Su último estudio de tomografía de emisión de positrones-tomografía axial computarizada (PET-CT) mostró datos en relación con enfermedad estable.

Palabras clave: Krukenberg, metástasis, colon, ovario.

\section{Abstract}

The Krukenberg tumor is a fibroma of digestive origin and presents metastasis to ovary, the highest incidence its between 30 and 40 years. Malignant tumors of the ovary of 3 to $6 \%$ correspond to metastatic tumors. The rapid growth is the main characteristics of this, approximately 60 to $80 \%$ occur bilaterally. Microscopically presented mucous material wealth in the form of seal ring. Adenocarcinoma of the colon is the tumor that mostly simulates primary carcinoma of ovary; from 10 to $30 \%$ of women with carcinoma of colon will present a secondary tumor focus in ovary. Case report: Female 41 years with generalized abdominal pain and vomiting, performs ultrasound finding $11 \mathrm{~cm}$ left ovary-dependent mass. Laparoscopy showing green and abundant liquid in cavity, peritoneal implants in bladder, SAC and omentum; liquid and node in omentum is taken, they are sent and it reports metastatic adenocarcinoma. We proceed to laparotomy for cytoreduction. Report definitive histopathological primary moderately differentiated adenocarcinoma of the colon metastatic to ovary, "Krukenberg tumor". Today patient continues treatment with oncology-based chemotherapy, latest PET-CT study with data in connection with stable disease.

Keywords: Krukenberg, metastasis, colon, ovary.
* Médico Cirujano, Residente de la Especialidad en Ginecología y Obstetricia del Hospital Ángeles México.

₹ Médico Cirujano, Especialista en Ginecología y Obstetricia, Cirugía Laparoscópica. Médico adscrito al Hospital Ángeles México.

$\S$ Médico Cirujano, Especialista en Ginecología y Obstetricia. Médico adscrito al Hospital San Ángel Inn.

Ciudad de México, México.
Correspondencia:

Dr. Carlos Enrique Soto Aburto

Correo electrónico: charly_389@hotmail.com

Aceptado: 08-11-2019.

www.medigraphic.com/actamedica

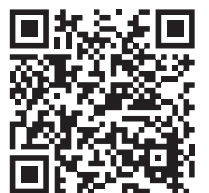




\section{INTRODUCCIÓN}

El tumor de Krukenberg fue descrito por primera vez en el año de 1896 por Friedrich Krukenberg, quien lo detalla como un fibroma mucosecretor, caracterizado por células en forma de anillo de sello. ${ }^{1}$

La mayor incidencia de este tumor se produce en pacientes entre 30 y 40 años, sin embargo, también se ha observado entre las pacientes postmenopáusicas. ${ }^{1} \mathrm{El}$ ovario es el sitio más común en el que ocurre metástasis de cáncer, el cual es de origen digestivo. ${ }^{2}$ Del total de los tumores malignos del ovario, un 3 a $6 \%$ de éstos corresponde a tumores metastásicos. ${ }^{3} \mathrm{El}$ crecimiento rápido es una de las principales características de este tumor, además aproximadamente el 60 a 80\% de los casos se presenta de manera bilateral. ${ }^{4}$

Macroscópicamente, estos tumores suelen ser sólidos, lisos y esféricos, aunque pueden presentarse en forma nodular; son de consistencia firme, gelatinosa o esponjosa y no suelen estar adheridos a otras estructuras. En promedio miden $10 \mathrm{~cm}$, aunque pueden llegar a medir hasta $20 \mathrm{~cm}^{4}$

Microscópicamente se pueden ver células epiteliales mucíparas. Las células mucosecretoras pueden presentarse en pequeñas o grandes agrupaciones ovales o redondeadas con citoplasma pálido y vacuolado y con material mucoso abundante en forma de anillo de sello. ${ }^{5}$

La mayoría de las pacientes que presentan metástasis hacia el ovario suelen ser más jóvenes que las que presentan un tumor primario sin que afecte el ovario. ${ }^{6}$

Existen distintas vías de diseminación de la enfermedad: la vía linfática y la vía hematógena son las principales en estos casos. $^{7}$

Todos los tumores de ovario tanto metastásicos como primarios suelen evolucionar de manera silenciosa hacia el abdomen sin generar dolor $u$ otra sintomatología agregada. En el momento en que la tumoración alcanza un volumen considerable es cuando, generalmente, se vuelve sintomática. Está descrito que hasta el 90\% de las pacientes pueden presentar sintomatología ovárica o de compresión hacia otros órganos, sin embargo, puede ser que el único motivo de la consulta sea el aumento de volumen abdominal, o bien, que sea un hallazgo incidental. ${ }^{8}$

El adenocarcinoma de colon es el tumor que mayormente simula al carcinoma primario de ovario; se estima que del 10 al 30\% de las mujeres con carcinoma de colon presentará, en algún momento, focos tumorales secundarios en el ovario. ${ }^{9}$

\section{REPORTE DEL CASO}

Se presenta el caso de una mujer de 41 años, nuligesta, que refiere como la fecha de su última menstruación el día
15 junio de 2018. Cuenta con el antecedente de cirugía ocular sin complicaciones aparentes, realizada en 2016. El resto fue interrogado y negado.

Refiere que inició su padecimiento tres días previos a su ingreso con dolor abdominal generalizado tipo cólico, el cual era intermitente y de 4/10 en la escala numérica análoga de dolor (ENA); el dolor no presentaba irradiaciones. En una ocasión hubo vómito, motivo por el cual acudió con un médico tratante.

Se realizó un ultrasonido abdominal, el cual reportó lo siguiente: útero de morfología habitual, con dimensiones aproximadas de $7.7 \times 3.5 \times 4.5 \mathrm{~cm}$, patrón miometrial aparentemente homogéneo y un espesor de $4.8 \mathrm{~mm}$. En el anexo derecho persistió una estructura ovalada hipoecoica con patrón folicular conservado, borde liso y dimensiones de $3.3 \times 1.8 \times 3.1 \mathrm{~cm}$ con un volumen de $10.2 \mathrm{~cm}^{3}$. También se reportó una masa que se extiende desde la fosa iliaca izquierda hasta la cicatriz umbilical; es de bordes parcialmente definidos, heterogénea, con zonas de mayor y menor ecogenicidad, así como anecoicas.

Al momento de la aplicación de Doppler, el color presenta vascularidad central y periférica con dimensiones aproximadas de $9.5 \times 9.4 \times 11 \mathrm{~cm}$. No se observa líquido libre en espacios peritoneales.

Se recabaron los estudios de laboratorio del 22 de junio de 2018, los cuales reportaron: hemoglobina ( $\mathrm{Hb})$ 11, hematocrito (HTO) de 32.6, plaquetas (PLT) 357, leucocitos (LEUC) 7.0, tiempo de protrombina (TP) 12.7, International Normalized Ratio (INR) 1.09 y tromboplastina parcial (TTP) 23.4.

Se realizó laparoscopia diagnóstica y se visualizaron asas de intestino distendidas y líquido libre de aspecto verde y abundante en cavidad; implantes peritoneales en vejiga, fondo de saco y epiplón, así como adherencias de intestino delgado a la pared abdominal y una tumoración en el ovario izquierdo de $11 \mathrm{~cm}$.

Se tomó una muestra de líquido peritoneal y de nódulo de epiplón, los cuales fueron enviados a estudio histológico transoperatorio que reportó un adenocarcinoma metastásico.

Se convirtió a laparotomía para realizar citorreducción y se observó la oclusión intestinal a nivel de yeyuno, por lo que se resecaron $15 \mathrm{~cm}$ del mismo y se realizó una resección entero-entero. También se resecó el epiplón, observando implante en colon transverso, por lo que se decidió resecar $20 \mathrm{~cm}$ de colon y se realizó una enteroentero anastomosis.

Se efectuó una histerectomía subtotal más una salpingooforectomía bilateral, encontrando fondo de saco y recto con datos de actividad tumoral. Finalmente se dio por terminado la cirugía sin más incidentes ni complicaciones.

Los hallazgos fueron los siguientes: tumoración en yeyuno y colon transverso, salpinge y ovario con tumoración en 
ovario izquierdo, útero con cérvix amputado con salpinge y ovario derecho.

La impresión diagnóstica histopatológica fue: segmento yeyunal y adenocarcinoma de tipo mucinoso invasor ulcerado con extensión a la pared e invasión a la serosa sin perforarla y bordes quirúrgicos de resección negativos; resección de colon y adenocarcinoma de tipo mucinoso, ulcerado y perforado, con extensión en todo el espesor de la pared e invasión a serosa y perforación de la misma, y bordes de resección negativos; epiplón con presencia de células neoplásicas malignas. El ovario izquierdo y el derecho tuvo presencia de adenocarcinoma de tipo mucinoso con invasión de la cápsula sin perforación de la misma.

Se dio seguimiento intrahospitalario durante 10 días a la paciente, quien evolucionó favorablemente; toleró la vía oral y presentó evacuaciones; el resto ocurrió sin complicaciones. Por esta razón, se decidió su egreso y se realizó una interconsulta de manera externa con el servicio de oncología para continuar con su tratamiento a base de quimioterapia.

Se recabó el estudio inmunohistoquímico con el diagnóstico de adenocarcinoma moderadamente diferenciado, primario de colon con expresión de citoqueratina 20, CDX2, SAT-B2 y villina. La paciente continuó con un tratamiento a base de quimioterapias con nueva inmunohistoquímica, la cual reportó una disminución de 10 mm en las dimensiones y metabolismo del engrosamiento nodular peritoneal, en la corredera parietocólica izquierda con metabolismo difuso con SUVmax (maximum standardized uptake values) de 2.28 (previo de $14 \mathrm{~mm}$ con SUVmax de 4.6).

Actualmente la paciente continúa en tratamiento oncológico basado en quimioterapia. Se realizó un estudio tipo PET-CT, que reportó engrosamiento nodular peritoneal en corredera parietocólica izquierda de $08 \mathrm{~mm}$ con SUVmax de 1.7 (previo $10 \mathrm{~mm}$ SUVmax de 2.2) y que persiste sin cambios. También reporta una imagen sólida que presenta realce heterogéneo con medio de contraste, adyacente a recto-sigmoides, con dimensiones de $29 * 24 \mathrm{~mm}$ con SUVmax de 3.8 (previo 26*24 mm con SUVmax de 3.9).

La impresión diagnóstica fue: datos en relación con enfermedad estable.

\section{CONCLUSIÓN}

Actualmente no se cuentan con estudios de tamizaje o de abordaje diagnóstico estandarizado ni siquiera en la literatura internacional, por lo que la mayoría de los casos se presentan como hallazgos incidentales en el tratamiento primario de otra patología o al momento de realizar alguna otra cirugía, como se presenta en este caso. Esto ha dado como resultado el diagnóstico de esta patología en etapas clínicas avanzadas $y$, por consiguiente, un mal pronóstico para la paciente.
En algunos casos es difícil distinguir si el tumor es un tumor primario de ovario o si es de origen metastásico. Estudios refieren que alrededor del $45 \%$ del total de los casos de cáncer de colon fue diagnosticado como tumor primario de ovario en primera instancia, tal como sucedió en el caso anteriormente presentado. ${ }^{10}$

Como podemos analizar, el reporte microscópico de este caso es similar a lo descrito en la literatura, es decir, se pudo observar componente mucinoso abundante, además de presentar marcadores CDX-2 en el estudio de inmunohistoquímica; éstos, al ser positivos, nos confirman que el tumor primario es de origen colónico.

La importancia de conocer si el tumor primario es de origen colónico u ovárico recae en algoritmo y estandarización son adecuados para su tratamiento, ya que actualmente se sabe que la cirugía de citorreducción puede ayudar a la supervivencia de las pacientes.

La presentación de este caso nos deja en claro que tenemos un mundo de oportunidades para desarrollar estudios de tamizaje de patologías ováricas, así como mejoras en el abordaje diagnóstico y en el estandarizado, además de un adecuado tratamiento multidisciplinario que se verá reflejado en la mejora de la calidad de vida de nuestras pacientes y en su pronóstico y supervivencia.

\section{REFERENCIAS}

1. Young RH. From Krukenberg to today: the ever present problems posed by metastatic tumors in the ovary: part l. Historical perspective, general principles, mucinous tumors including the Krukenberg tumor. Adv Anat Pathol. 2006; 13 (5): 205-227.

2. Mazur MT, Hsueh S, Gersell DJ. Metastases to the female genital tract. Analysis of 325 cases. Cancer. 1984; 53 (9): 1978-1984.

3. Blanco FJ, Hernando E, García JL, Cebollero MP, Bavai A, Del Río $\mathrm{F}$ et al. Tumor de Krukenberg. A propósito de un nuevo caso. Cir Española. 2000; 68: 68-70.

4. Prat J. Ovarian carcinomas, including secondary tumors: diagnostically challenging areas. Mod Pathol. 2005; 18 Suppl 2: S99-S111.

5. Jun SY, Park JK. Metachronous ovarian metastases following resection of the primary gastric cancer. J Gastric Cancer. 2011; 11 (1): 31-37.

6. Antila R, Jalkanen J, Heikinheimo O. Comparison of secondary and primary ovarian malignancies reveals differences in their pre- and perioperative characteristics. Gynecol Oncol. 2006; 101 (1): 97-101.

7. Yamanishi Y, Koshiyama M, Ohnaka M, Ueda M, Ukita S, Hishikawa $\mathrm{K}$ et al. Pathways of metastases from primary organs to the ovaries. Obstet Gynecol Int. 2011; 2011: 612817.

8. Kiyokawa T, Young RH, Scully RE. Krukenberg tumors of the ovary: a clinicopathologic analysis of 120 cases with emphasis on their variable pathologic manifestations. Am J Surg Pathol. 2006; 30 (3): 277-299.

9. Daya D, Nazerali L, Frank GL. Metastatic ovarian carcinoma of large intestinal origin simulating primary ovarian carcinoma. A clinicopathologic study of 25 cases. Am J Clin Pathol. 1992; 97 (6): 751-758.

10. López-Arias A, Salazar-Campos JE, Pérez-Montiel D, VillavicencioValencia V, Cantú de León D. Tumores metastásicos de ovario. Un reto diagnóstico y terapéutico. Gaceta Mexicana de Oncología. 2016; 15 (1): 47-51. 